\title{
Mental health outcomes for individuals with chronic hepatitis C infection.
}

\author{
William W. Thompson ${ }^{1}$, Mohammed A. Khan $^{1}$, Jay Soh ${ }^{1}$, Lauren Canary ${ }^{1}$, Michael Blank ${ }^{2}$, \\ Noele P. Nelson ${ }^{1}$ \\ ${ }^{1}$ National Center for HIV/AIDS, Viral Hepatitis, STD, and TB Prevention, US Centers for Disease Control \& Prevention, Atlanta, Georgia, United States, \\ ${ }^{2}$ UNIVERSITY OF PENNSYLVANIA - PERELMAN SCHOOL OF MEDICINE, Philadelphia, Pennsylvania, United States
}

Objective

Using data from the 2011-2015 IBM MarketScan ${ }^{\circledR}$ Commercial Claims and Encounters, we sought to assess the relationship between mental health outcomes and chronic hepatitis $\mathrm{C}$ infection after adjusting for important confounders. Persons with HCV antibody and RNA test results between 2011 and 2015 and continuous enrollment in fee-for-service plans were included in the analysis

\section{Introduction}

Hepatitis $\mathrm{C}$ virus (HCV) infection is a leading cause of liver disease-related morbidity and mortality in the United States and HCV incidence has been increasing. Mental illness may impact the likelihood of initial HCV infection, progress and adherence to treatment along the hepatitis $\mathrm{C}$ care cascade, and risk of subsequent reinfection for those cured of hepatitis $\mathrm{C}$. The relationship between HCV infection and mental illness is not well understood and many studies have lacked sufficient sample size to adjust for important confounders. We sought to explore the association between chronic HCV infection and mental illness after adjusting for important confounders.

\section{Methods}

We obtained data from the 2011-2015 IBM MarketScan® Commercial Claims and Encounters and Medicare Supplemental and Coordination of Benefits databases. These data consist of inpatient and outpatient service claims for persons with employersponsored health insurance coverage and their dependents.

Persons with HCV antibody and RNA test results between 2011 and 2015 and continuous enrollment in a fee-for-service plan were included in the analysis. Chronic HCV infection was defined by a positive HCV RNA test result. Controls without chronic HCV infection had a negative HCV antibody test result and no positive HCV antibody or RNA test result in the preceding or following year. The index date was defined by the date of the earliest positive HCV RNA or negative HCV antibody test. Demographic characteristics were obtained from the MarketScan ${ }^{\circledR}$ enrollment tables. All enrollees in the study population were at least 18 years old during the year of the index date. The analysis sample was restricted to persons who were identified as receiving outpatient prescription drug claims data feeds. We estimated adjusted odds ratios (OR) for the association between mental illness (ICD-9 code 295 or 296) and HCV RNA status. Multivariate models included age (18-44, 45-64, 65+ years), sex, region, and an adjusted Charlson Comorbidity Index which excluded liver disease and hepatocellular carcinoma.

\section{Results}

We identified 2,847 individuals with chronic HCV infection (HCV RNA+) and 57,418 controls who were HCV antibody negative. With respect to age, $83 \%$ of HCV RNA+ individuals were aged 45-64 years while only $43 \%$ of the HCV antibody negative individuals were in the same age range. Similarly, for sex, $62 \%$ and $40 \%$ of HCV RNA+ individuals and controls, respectively, were male. For unadjusted analyses, age, sex, region, comorbid conditions, and mental illness (OR=2.25 [95\% CI; 1.52 - 3.34]) were all statistically associated with HCV RNA+. For the multivariate adjusted models, these same variables remained statistically significant. For the multivariate model, individuals with a mental illness were more likely to be HCV RNA+ relative to HCV antibody negative controls. $(\mathrm{OR}=1.95[95 \% \mathrm{CI} ; 1.30-2.93])$. 


\section{Conclusions}

This study demonstrated a strong association between mental illness and HCV chronic infection after adjusting for important confounders including other comorbid conditions. A growing body of research suggests that persons with mental illness are at increased risk for contracting and transmitting HCV due to high rates of substance use and high-risk sexual behavior among infected persons as well as high rates of sexual victimization. HCV prevention efforts should be directed toward individuals with mental illness or seeking treatment for mental illness. 